\title{
Incidence of symptoms and AIDS in 146 Swedish haemophiliacs and blood transfusion recipients infected with human immunodeficiency virus
}

\author{
Johan Giesecke, Gianpaolo Scalia-Tomba, Ove Berglund, Erik Berntorp, Sam Schulman, \\ Lennart Stigendal
}

\begin{abstract}
The times from infection with the human immunodeficiency virus (HIV) to the onset of the first clinical symptom and the development of AIDS were studied prospectively in 98 haemophiliacs and 48 blood transfusion recipients infected with the virus. Patients were followed up for a median of 61 months after infection, the dates of infection being either known exactly or estimated from the interval between the last negative and first positive HIV antibody test result. The rate of progression to AIDS was significantly higher for the transfusion recipients than for the haemophiliacs. The difference in time to the occurrence of the first clinical symptom was less pronounced between the two groups, though pointing in the same direction.

The results suggest that on average roughly half of all patients positive for HIV will develop some clinical sign or symptom within five to six years after infection.
\end{abstract}

\section{Introduction}

The incubation time from infection with the human immunodeficiency virus (HIV) to the development of AIDS is a factor of crucial epidemiological importance. Any prognosis of the course of the epidemic requires knowledge of this value. As with any other infectious disease, however, it is misleading to talk about the incubation time as if it were a constant; instead we should try to describe its distribution - that is, the proportion of infected patients who have developed AIDS as a function of time since infection. Estimates of the distribution of the incubation time of AIDS must rely on studies of patients with known dates of infection. Longitudinal surveys of cohorts of homosexual men, ${ }^{1}$ haemophiliacs, ${ }^{2}$ and blood transfusion recipients $\mathrm{s}^{3}$ are beginning to yield information about the incidence of AIDS up to eight years after infection.

Most published attempts to estimate the distribution of incubation times have been based on data from patients with AIDS in the United States who have contracted HIV from blood transfusions. ${ }^{4-7}$ These estimates have been used to make predictions about the future size of the AIDS epidemic. ${ }^{89}$ There is one serious problem with this approach: the original subjects described by Peterman et al were all patients who already had a diagnosis of AIDS. ${ }^{10}$ Nothing is known about the number of people infected by blood transfusions who have not yet developed the disease. As we have only a limited study period, excluding these patients will lead to an underestimate of the average incubation period. Several workers have tried to compensate for this lack of information by assuming some given type of distribution for the incubation time in the entire infected population. They have then fitted this assumed distribution to the observed data to find its probable shape. There is, however, very little firm support for any of these distributions.

In this study we collected data on all Swedish haemophiliacs infected with HIV as well as on all infected recipients of blood from a group of Swedish donors positive for the virus. These 146 patients were closely followed up and the times of appearance of symptoms related to HIV, diagnosis of AIDS, and death recorded. From these complete data it should be possible to obtain a better estimate of the distribution of the incubation time.

\section{Patients and methods} HAEMOPHILIACS

There are about 350 known haemophiliacs who require regular or on demand infusions of coagulation factor concentrates in Sweden. They are all taken care of at one of three centres in Stockholm, Malmö, or Gothenburg. Beginning in 1984, this entire group was screened for antibodies to HIV. They were also tested regularly after heat treated concentrate was introduced for all haemophiliacs in 1985. At the end of 1985 four patients with haemophilia B received concentrate treated with hydrophobic gel but unheated, which turned out to be infective. They were assessed as seropositive in early 1986. Apart from these, no haemophiliacs seroconverted after 1985.

Exactly 100 of these 350 patients were found to be positive for HIV antibody, and 98 of these are included in the statistics below. Data for the two missing patients were incomplete, but neither had developed AIDS. Forty five of the infected haemophiliacs were being or had been treated at the Stockholm centre, 43 in Malmö, and 12 in Gothenburg. The patients from the first two centres have been described elsewhere. ${ }^{11} 12$ Patients in this study had no other known risk factors for HIV infection. There was no evidence that any haemophiliac in Sweden had died of any HIV related illness before the entire group was screened.

All imported factor concentrate given in Stockholm and Malmö has been heat treated since 1983, and in Gothenburg since 1985. Swedish factor VIII concentrate has been heat treated since 1985 and factor IX concentrate since 1987.

\section{TRANSFUSION RECIPIENTS}

Screening of blood donors in Sweden began in the spring of 1985 and was complete by the autumn of that year. In 1986 a special attempt was made to find HIV infected blood transfusion recipients in Stockholm. Thirty five known HIV positive patients reported having been blood donors before their antibody state was discovered. From the complete files of transfusions in Stockholm 349 recipients of blood from these donors were found, of whom 180 were alive at the time of study. These recipients were asked to come to a clinic for HIV testing and counselling, starting with the recipients who had had the last transfusion from each donor and working backwards. All but one of the recipients who were asked to volunteer for testing agreed to come.

In this group of recipients, as in the group described by Ward $e t a l,{ }^{13}$ it soon turned out that once a donor had infected someone all subsequent transfusions led to infection. The backward tracing of recipients was thus stopped when two consecutive recipients of any one donor turned out to be antibody negative. In this way we found that 21 of the HIV positive donors had not infected anyone; thus presumably they had become infected after giving up donating blood. Fourteen of the donors had infected a total of 50 living recipients, of whom 48 are included below. Of the other two, one was
Correspondence to: Dr Giesecke. 
living abroad and one had died of causes not related to HIV. Patients included in the study had no other known risk factors for HIV infection.

That every transfusion from an antibody positive donor leads to infection is illustrated by the following. For one donor one of the recipients was found to be antibody negative, whereas several previous and subsequent recipients were antibody positive. Careful review of this patient's medical chart showed that the unit of blood had been ordered from the blood bankand was thus registered as a transfusion-but had never been given to the patient on the ward. We may therefore surmise that 33 of the 169 recipients who had died before the study were also infected and that a further 25 patients were possibly infected. Most of these patients had died within six months after the transfusion. One of these 58 patients apparently died of AIDS. A more detailed account of the transfusion recipients will be published elsewhere. ${ }^{14}$

\section{DEFINITION OF EVENTS}

The health state of each patient in May 1987 (haemophiliacs) or July 1987 (transfusion recipients) was assessed. For those patients who had clinical signs of HIV infection the date of appearance of the first such sign was recorded. Likewise, dates of the diagnosis of AIDS and death were recorded where applicable. The decision on which symptoms should be regarded as the first clinical sign of HIV infection was difficult, but our intention was to include any symptom that probably would have induced an unknowingly infected person to seek medical advice. This definition has obvious clinical value. These symptoms included lymphadenopathy syndrome, mycosis of nails, diarrhoea, oral candidiasis, herpes zoster, prolonged fever, weight loss, symptomatic thrombocytopenia, and AIDS. Acute HIV infection was diagnosed retrospectively in a few cases, but as its symptoms are so non-specific neither it nor abnormal laboratory findings were counted as "first symptom" in the calculations below.

\section{STATISTICAL ANALYSIS}

Incubation times were analysed according to the Kaplan-Meier survival curve technique. For transfusion recipients dates of infection were known exactly. For haemophiliacs there was usually uncertainty about the date of infection, and we therefore estimated this from the interval between the last negative and first positive HIV results (in cases in which the first test gave a positive result we assumed that the earliest possible date of infection was January 1978). The date of seroconversion was estimated as the midpoint of this interval. (An alternative way of estimating time of infection is discussed below.) Differences between the estimated distributions of incubation times and associations between incubation times and age were analysed by the log rank and generalised Wilcoxon tests, the first being sensitive to differences in the tails of the distributions, the second giving more weight to differences for shorter times (see, for example, Cox and Oakes ${ }^{15}$ ). Incubation times to clinical symptoms and AIDS were analysed separately. Statistical analyses were performed with PROC LIFETEST and PROC LIFEREG in SAS (Statistical Analysis System, SAS Institute Inc, Cary, North Carolina).

\section{Results}

Table I shows the estimated or known years of seroconversion for all patients in the series. The median follow up time for the 98 haemophiliacs was 70 months. Symptoms were recorded in 39 of these patients, of whom seven developed AIDS. In the
TABLE I-Estimated years of seroconversion among haemophiliacs and recipients of infective transfusions

\begin{tabular}{lrrrrrrrrrr}
\hline & \multicolumn{1}{c}{ Year } \\
\cline { 2 - 7 } & 1979 & 80 & 81 & 82 & 83 & 84 & 85 & 86 \\
\hline No of haemophiliacs & 11 & 21 & 36 & 15 & 11 & 1 & 2 & 1 \\
No of transfusion recipients & 0 & 0 & 0 & 10 & 13 & 20 & 5 & 0
\end{tabular}

transfusion recipients the median follow up time was 43 months. Sixteen of these 48 patients developed symptoms, of whom 10 progressed to AIDS.

Figures 1 and 2 show the Kaplan-Meier curves for the times to occurrence of symptoms and diagnosis of AIDS. Other results of statistical analysis are reported and commented on below.

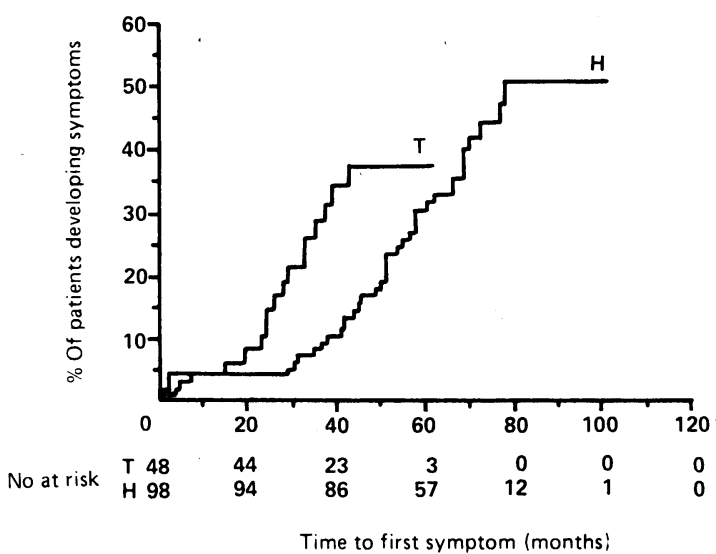

FIG 1-Proportions of infected patients who developed symptoms as function of time since infection. $\mathbf{T}=$ Transfusion recipients. $\mathbf{H}=$ Haemophiliacs

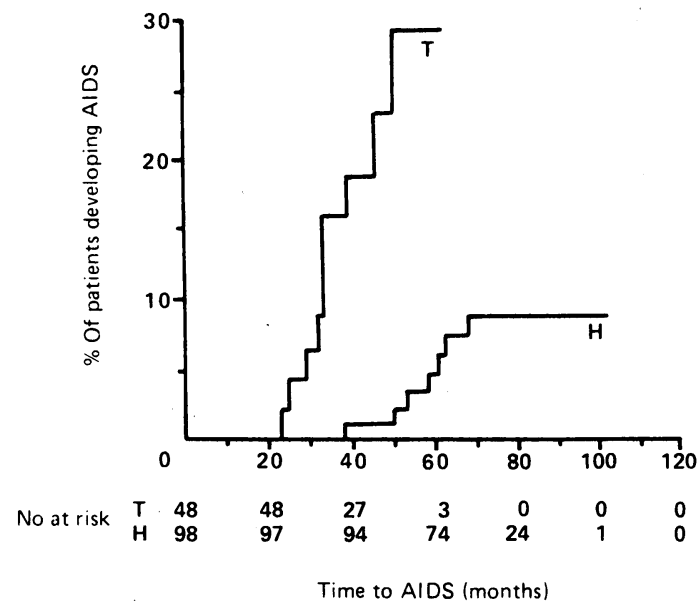

FIG 2-Proportions of infected patients who developed AIDS as function of time since infection. $\mathbf{T}=$ Transfusion recipients. $\mathbf{H}=$ Haemophiliacs

\section{Discussion}

\section{TIME TO FIRST SYMPTOM}

There was a significant difference in time to first symptom between the two groups ( $\log$ rank test, $\mathrm{p} \approx 0.02$; Wilcoxon test, $\mathrm{p}<0.01$ ), transfusion recipients developing symptoms earlier than haemophiliacs. Estimating visually from figure 1, we conclude that on average roughly half of HIV infected patients will develop some symptom of their infection that should induce them to seek medical advice after five or six years. We admit that including lymphadenopathy among the symptoms that may be experienced by the patient is debatable and probably dependent on the patient's self perceived risk of HIV infection. Including the lymphadenopathy syndrome may in some respect yield too high an incidence of 
symptoms but, on the other hand, as more physicians become acquainted with the acute retroviral syndrome the proportion of infections that are detected early ought to increase.

\section{INCUBATION TIME TO AIDS}

There was a significant difference (log rank test, $\mathrm{p}<0.001$; Wilcoxon test, $\mathrm{p}<0.001)$ in the incidence of progression to AIDS between the haemophiliacs and transfusion recipients (fig 2). Among the haemophiliacs none had developed AIDS three years after seroconversion compared with $1 \%$ at four years and $5 \%$ at five years. Of the transfusion recipients, $16 \%$ had AIDS after three years, $23 \%$ after four years, and $29 \%$ after five years.

Table II summarises some of the studies on incubation times among different groups of patients infected with HIV. Compared with the series of Eyster et $a l^{2}$ our haemophiliacs showed a lower incidence of progression to AIDS at four to six years. Our transfusion recipients, however, had a higher incidence of AIDS than reported by Ward et al, ${ }^{16}$ though the groups studied were small. Medley et al calculated the distribution of incubation times among transfusion recipients from a number of known cases of AIDS and estimates of the total number of people infected in this way. ${ }^{5}$ They predicted only a $20 \%$ cumulative incidence after five years, but there was rather large uncertainty in their estimate of the denominator for relative incidence.

TABLE II-Estimated cumulative percentage incidences of AIDS in different groups of patients (SE in parentheses)

\begin{tabular}{lccccccc}
\hline & \multicolumn{8}{c}{ Years from known or estimated time of infection or } \\
seroconversion
\end{tabular}

\section{HETEROGENEITY OF INCUBATION TIMES}

Several investigators have reported that the incubation time among transfused children seems shorter than among adults. Our series of transfusion recipients included only two children, neither of whom developed any sign of HIV infection. The average age of the transfusion recipients was 55 and there was no significant difference in the rate of progression to AIDS between the older and younger patients. There was also no obvious relation between age at infection and time to the occurrence of AIDS in haemophiliacs. In this group, however, there was a significant difference among patients treated at the different centres, those in Gothenburg progressing to AIDS sooner than at the two other centres. This may be an effect of the different types and sources of factor concentrate used, as explained above. Such a difference for haemophiliacs treated at different centres was also reported by Eyster et al. ${ }^{2}$

Though statistical tests failed to show any relation between age at infection and incubation time, it is interesting that the average age in the haemophiliac groups from the treatment centres with lowest incidence was about 30 compared with 35 in the Gothenburg group and 55 in the transfusion recipients. Hence age may possibly play a part in incubation time, which could not be detected in our series because of the small sample size and limited number of cases (17 cases of AIDS among 148 patients).

More interestingly, of the 10 transfusion recipients who progressed to AIDS during the study, six were infected by the same donor. Blood from this man, however, had been given to 15 of the 48 patients. It is difficult to assess the relevance of this but possibly he carried an especially virulent strain of HIV or had persistently high virus titres.

\section{STATISTICAL ASPECTS}

\section{Estimation of time of infection}

In this series, as in other cohort studies of haemophiliacs and people infected sexually, there was often uncertainty about the time of infection. The usual remedy is to estimate this as the midpoint of the interval between the last negative (or, if this is unknown, a reasonable first possible such date) and the first positive antibody test result. We followed this practice for comparability. This method, however, implicitly presupposes that the risk of infection has been constant during the period including the possible times of infection, in contrast with the commonly held view that the number of infected blood donors-and hence the risk of infection from blood products-has been rising exponentially during the same period. This reasoning was adopted by Taylor et al, who assumed that the risk of infection was increasing linearly on the uncertainty intervals and used this assumption in their estimation of incubation times. ${ }^{17}$ The hypothesis of exponential increase leads to the following formula for the estimated time of infection: estimated month of infection $=\left(b-a e^{-\beta(b-a)}\right) /\left(1-e^{-\beta(b-a)}\right)-1 / \beta$, where $b$ denotes the month of the first positive HIV test result, a the month of the last negative HIV test result, and $\beta$ equals $(\ln 2) / 12=0.058$. This choice of $\beta$ corresponds to a doubling time of one year for the risk of infection.

In our data on haemophiliacs we found that the average interval of uncertainty was about three years and that in 39 subjects the first antibody test result was positive. Most of these tests, however, were performed before 1984. It may be noted that the "exponential" estimate is always closer to the right endpoint of the uncertainty interval than is the midpoint.

The effect of the two methods of estimating time of infection on the subsequent analysis of incubation time before the onset of AIDS was slight owing to the relatively low values achieved by the cumulative incidence in this study, and the highly significant difference between the two groups of patients remained. The effect was seen more easily in the analysis of time to the occurrence of symptoms, where, for example, the $30 \%$ quantile in the distribution for haemophiliacs was estimated to be 58 months by the midpoint method and 50 months by the exponential method. Furthermore, the significance of the difference between the subject groups disappeared (log rank test, $p \approx 26 \%$; Wilcoxon test, $p \approx 8 \%$ ), though the visual relation between the transfusion recipients and the haemophiliacs remained, the transfusion recipients having symptoms sooner. Thus the difference between the two groups of patients in time to the occurrence of the first clinical symptom was less well established than the difference in time to the onset of AIDS. This seems to be relatively important in the estimation procedure.

Furthermore, when infection transmitted by infusion is compared with other modes of transmission we must remember that in the first case the actual time from infection is measured, whereas in the second the issue is the time from seroconversion, though many authors, ourselves included, often use these words interchangeably. The difference is probably negligible in most instances. 
Bias in resulting estimate of distribution

It is perhaps more surprising that any method of estimating the time of infection by a single time point will result in the estimation of a biased distribution of incubation time. The exact resulting perturbation of the distribution is difficult to predict but plainly, for example, the variance will be overestimated, as the times subject to analysis will have one variance component in themselves (the natural variability of incubation times) and one additional variance component from the estimation of the time of infection. This second source of variance must of necessity be greater than zero, as the true times of infection are unknown. Taylor et al tried to correct for this effect, ${ }^{17}$ but we believe that the resulting distribution remains biased. We do not, however, know of any other method of correction.

\section{Fitting of distributions}

It is tempting to try to fit known distributional forms to data in order to stabilise estimates and be able to make predictions about the future course of infection. We are not convinced about the soundness of this approach. There is at present no way of choosing the correct distribution, and knowing or being able to estimate the left tail of the incubation times (in this study we barely reached the $10 \%$ level in the cumulative incidence of AIDS) does not carry much information about the central parts of this distribution.

We have checked the applicability of the often used Weibull distribution ${ }^{45}$ as a description of the present data by graphical methods-plotting estimated log cumulative hazard against log time, a plot that should yield approximately straight lines if the Weibull distribution were appropriate-and found that time to the onset of AIDS could be thus described but not time to the occurrence of first symptom. The parametric fitting of Weibull distributions to the haemophiliac and transfusion recipient groups separately, for example, resulted in estimates of the corresponding median incubation times before the onset of AIDS, which were $13 \cdot 4$ (SE 2) and $5 \cdot 7$ (1) years, respectively.
This work has been supported by the Swedish Medical Research Council (grant K87-27H-08175-01) and the Stockholm County Council.

1 Hessol NA, Rutherford GW, O'Malley PM, et al. The natural history of human immunodeficiency virus infection in a cohort of homosexual and bisexual men: a 7-year prospective study [Abstract]. Proceedings of third international conference on AIDS. Washington, DC. US Department of Health and Human Services, 1987:1.

2 Eyster ME, Gail MH, Ballard JO, Al-Mondhiry H, Goedert JJ. Natural history of human immunodeficiency virus infections in hemophiliacs: effects on T-cell subsets, platelet counts and age. Ann Intern Med 1987;107:1-6.

3 Peterman TA, Holmberg SD, Lui K-J. Transfusion-associated AIDS in the United States [Abstract]. Praceedings of third intermational conference on
Unterman TA, Holmberg SD, Lui K-J. Transfusion-associated AIDS in the United States [Abstract]. Proceedings of third international conference on
AIDS. Washington, DC: US Department of Health and Human Services, AIDS. W

4 Lui K-J, Lawrence DN, Morgan WM, Peterman TA, Haverkos HW, Bregman DJ. A model-based approach for estimating the mean incubation period of transfusion-associated acquired immunodeficiency syndrome. Proc Natl Acad Sci USA 1986;83:3051-5.

5 Medley GF, Anderson RM, Cox DR, Billard L. Incubation period of AIDS in patients infected via blood transfusion. Nature 1987;328:719-21.

6 Iversen O-J, Engen S. Epidemiology of AIDS-statistical analyses. f Epidemiol Community Health 1986:41:55-8.

7 Rees M. The sombre view of AIDS. Nature 1987;326:343-5.

8 Brookmeyer R, Gail MH. Minimum size of the acquired immunodeficiency syndrome (AIDS) epidemic in the United States. Lancet 1986;ii:1320-2.

9 van Druten JAM, de Boo Th, Jager JC, Heisterkamp SH, Coutinho RA, Ruitenberg EJ. AIDS prediction and intervention. Lancet 1986;i:852-3.

10 Peterman TA, Drotman DP, Curran JW. Epidemiology of the acquired immunodeficiency syndrome (AIDS). Epidemiol Rev 1985;7:1-21.

11 Schulman S, Biberfeld G, Blombäck $M$, et al. HIV infection in a defined population of Swedish haemophiliacs. Scand f Infect Dis 1987;19:159-66.

12 Berntorp E, Hansson BG, Böttiger B, et al. HIV seroconversion in Swedish haemophiliacs: relation to type and dosage of factor concentrate. European fournal of Haematology 1987;38:256-60.

13 Ward JW, Deppe DA, Samson S, et al. Risk of human immunodeficiency virus infection from blood donors who later developed the acquired immunodeficiency syndrome. Ann Intern Med 1987;106:61-2.

14 Berglund O, Beckman S, Grillner L, et al. HIV transmission by blood transfusions in Stockholm 1979-1985: nearly uniform transmission from infected donors. AIDS (in press).

15 Cox DR, Oakes D. Analysis of survival data. London and New York: Chapman and Hall, 1984

16 Ward JW, Deppe D, Perkins H, Kleinman S, Holland P, Allen J. Risk of disease in recipients of blood from donors later found infected with human immunodeficiency virus (HIV) [Abstract]. Proceedings of third international conference on AIDS. Washington, DC: US Department of Health and Human Services, 1987:2.

17 Taylor JMG, Schwartz K, Detels R. The time from infection with human immunodeficiency virus (HIV) to the onset of AIDS. $f$ Infect $D$ t 1986;154:694-7.

18 Goedert JJ, Landesman SH, Eyster ME, Biggar RJ. AIDS incidence in pregnant women, their babies, homosexual men and hemophiliacs [Abstract]. Proceedings of third international conference on AIDS. Washington, DC: US Department of Health and Human Services, 1987:71.

(Accepted 12 April 1988)

\title{
Adhesive Escherichia coli in inflammatory bowel disease and infective diarrhoea
}

\author{
D A Burke, A T R Axon
}

\section{Abstract}

The clinical features of ulcerative colitis and Crohn's disease are similar to those of infections of the bowel, although their cause is uncertain. Many bacteria that cause intestinal diseases adhere to the gut mucosa, and adhesion of pathogenic Escherichia coli is resistant to D-mannose. The adhesive properties of isolates of $\mathbf{E}$ coli were assessed by assay of adhesion to buccal epithelial cells with mannose added. The isolates were obtained from patients with inflammatory bowel diseases ( 50 with a relapse of ulcerative colitis, nine with ulcerative colitis in remission, 13 with Crohn's disease, and 11 with infectious diarrhoea not due to E coli) and 22 controls.

The median index of adhesion to buccal epithelial cells (the proportion of cells with more than $\mathbf{5 0}$ adherent bacteria) for $E$ coli from patients with ulcerative colitis in relapse was significantly higher $(43 \%)$ than that for controls $(5 \%)$ and patients with infectious diarrhoea (14\%). The index was not significantly different among isolates from patients with ulcerative colitis in relapse, Crohn's disease $(53 \%)$, and ulcerative colitis in remission (30\%). If an index of adhesion of $>25 \%$ is taken as indicating an adhesive strain $86 \%$ of isolates of $E$ coli from patients with inflammatory bowel disease were adhesive compared with $27 \%$ from patients with infective diarrhoea and none from controls.

The adhesive properties of the isolates from patients with inflammatory bowel disease were similar to those of pathogenic intestinal $\mathbf{E}$ coli, raising the possibility that they may have a role in the pathogenesis of the condition; the smaller proportion of adhesive isolates in patients with infective diarrhoea due to other bacteria suggests that the organism may be of primary importance rather than arising secondarily.

\section{Introduction}

Mucosal adhesion is a virulence factor that is expressed by pathogenic Escherichia coli in the intestine. Enterotoxigenic strains of $E$ coli are recognised 